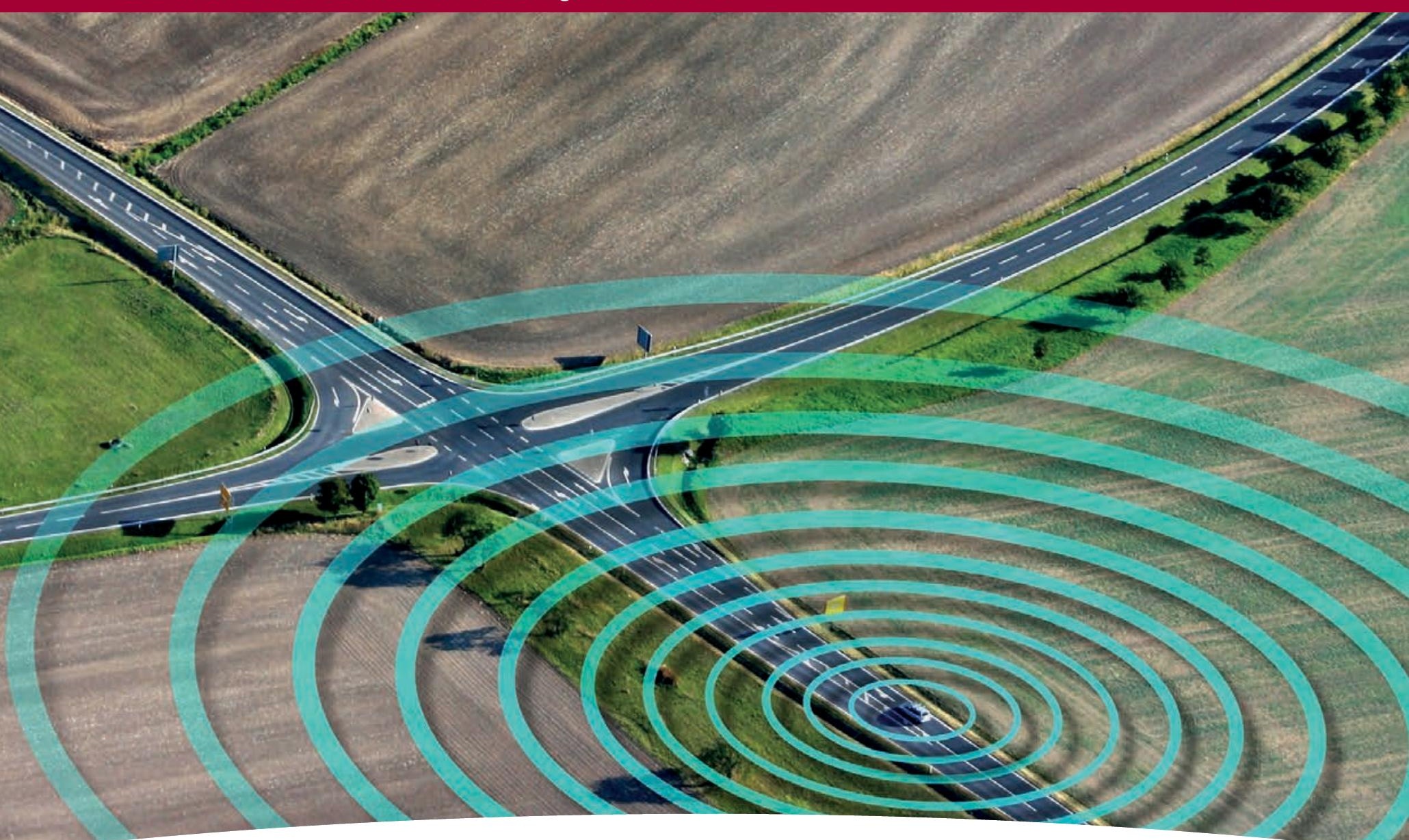

Unfallforschung kompakt

\title{
Verbesserung der Sicherheit durch Kommunikation zwischen Fahrzeug und Straße
}


Impressum

Gesamtverband der Deutschen Versicherungswirtschaft e. V.

Unfallforschung der Versicherer

Wilhelmstraße 43/43G, 10117 Berlin

Postfach 0802 64, 10002 Berlin

unfallforschung@gdv.de

www.udv.de

Redaktion: Dipl.-Ing. Thomas Heinrich, Dipl.-Ing. Jörg Ortlepp, Dipl.-Geogr. M.Sc. Jürgen Schmiele, Dipl.-Ing. Heiko Voß Layout: Michaela Gaebel

Technik: Monika Kratzer-Butenhof

Bildnachweis: Unfallforschung der Versicherer, TRANSVER GmbH

Erschienen: 10/2011 


\section{Vorbemerkung}

Seit 1970 sind die absoluten Zahlen der in Deutschland im Straßenverkehr getöteten und verletzten Personen rückläufig. Nicht zuletzt haben Verbesserungen bei der Fahrzeugsicherheit dazu geführt, dass die Schwere der Unfälle in den letzten Jahrzehnten kontinuierlich reduziert wurde. Inzwischen wird der Fahrer von einer Vielzahl von Sicherheitssystemen unterstützt. Dazu gehören neben den wohl bekanntesten wie ABS und ESP auch Systeme wie Spurverlassenswarner, Bremsassistenten oder Totwinkelwarner. Einen weiteren Sicherheitsgewinn erhofft man sich von Systemen, bei denen die Fahrzeuge miteinander kommunizieren (Vehicle to Vehicle) oder Informationen mit dem Straßenumfeld austauschen (Vehicle to Infrastructure). Dadurch sollen Gefahren frühzeitig erkannt und mitgeteilt werden, um dem Fahrer oder dem Fahrzeug eine rechtzeitige Reaktion zu ermöglichen. Dazu ist es notwendig zu wissen, wie und wo die relevanten Unfälle geschehen, welche Gemeinsamkeiten sie aufweisen und welche Informationen erforderlich sind, um auf die bevorstehende Gefahr hinzuweisen.

Um das herauszufinden wurde in dieser Untersuchung zunächst das Unfallgeschehen auf Landstraßen analysiert und daraus allgemeine Anforderungen an geeignete Assistenzsysteme abgeleitet. Es zeigte sich, dass infrastrukturbasierende Assistenzsysteme nicht zuletzt aufgrund des hohen technischen und finanziellen Aufwands nur in wenigen Anwendungsfällen wirklich sinnvoll erscheinen. 


\section{Inhalt}

$\begin{array}{ll}\text { Einleitung } & 4\end{array}$

2 Ergebnisse der Unfall-Fahrerassistenzsystem-Cluster

$2.1 \quad$ Cluster - Übersicht

2.2 Ausgewählte Cluster

2.2.1 Unfälle an Knotenpunkten

2.2.2 Fahrunfälle in Kurven und auf Geraden mit unangepasster Geschwindigkeit

2.2.3 Unfälle mit Gegenverkehr

2.2.4 Unfälle mit Fußgängern und Radfahrern im Längsverkehr

2.3 Zielführende Lösungswege für

Fahrerassistenzsysteme

$3 \quad$ Ergebnisse und Zusammenfassung 


\section{$1 \quad$ Einleitung}

Seit ihrem Höchststand im Jahr 1970 sind die absoluten Zahlen der in Deutschland im Straßenverkehr getöteten und verletzten Personen trotz gestiegener Fahrleistung rückläufig ( $\mathrm{Ab}$ bildungen $1 a, 1 b$ ). Dies gilt auch für Landstraßen. Jedoch sind Unfälle auf Landstraßen im Durchschnitt deutlich folgenschwerer als auf Autobahnen und innerhalb von geschlossenen Ortschaften (Abbildung 1c).

Deshalb sollten vor allem diese Unfälle bekämpft werden.

Die finanziellen Möglichkeiten der öffentlichen Hand sind jedoch begrenzt. Daher ist eine möglichst kostengünstige und effektive Ergänzung zu baulichen Maßnahmen wünschenswert.
Eine Möglichkeit könnten Fahrassistenzsysteme (FAS) sein, die auf Vehicle to Infrastructure-Kommunikation (V2I) basieren. Gründe für die derzeit geringe Verbreitung sind, dass die meisten Anwendungen sich noch in der Entwicklung befinden und dass viele Fragen bezüglich Wirksamkeit, Standardisierung, Betrieb, Haftung und Finanzierung ungeklärt sind.

Die Unfallforschung der Versicherer (UDV) beauftragte daher die TRANSVER GmbH München mit der Untersuchung, inwieweit V2Ibasierte Fahrerassistenzsysteme zu einer Verbesserung der Verkehrssicherheit auf Landstraßen geeignet sind. Fahrzeugautarke und auf der Kommunikation von Fahrzeug zu Fahrzeug (V2V) basierte Fahrerassistenzsysteme wurden bei der Untersuchung als alternative Lösungswege berücksichtigt.

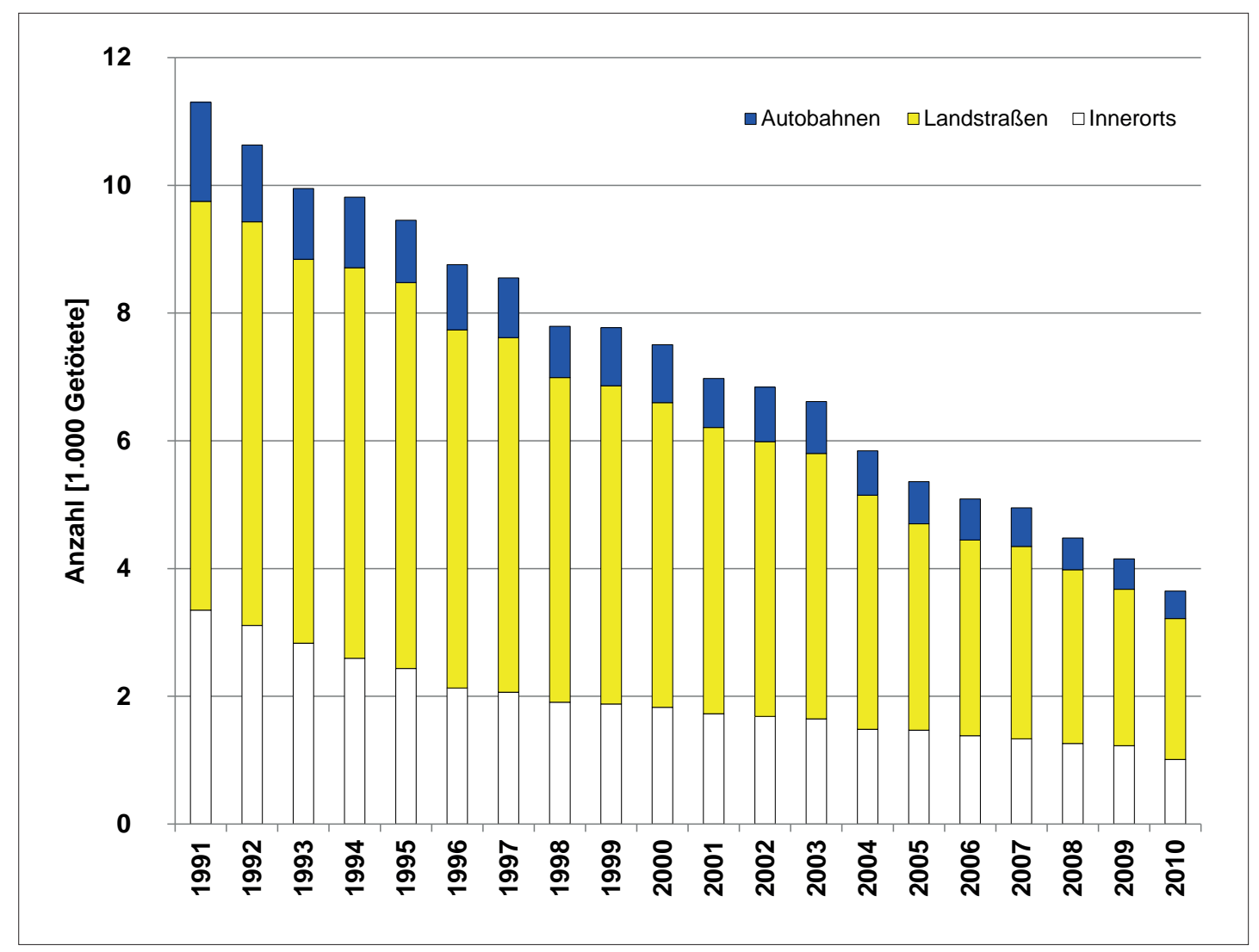

Abbildung 1a:

Entwicklung von schweren Personenschäden (Getötete) nach Ortslage seit 1991 


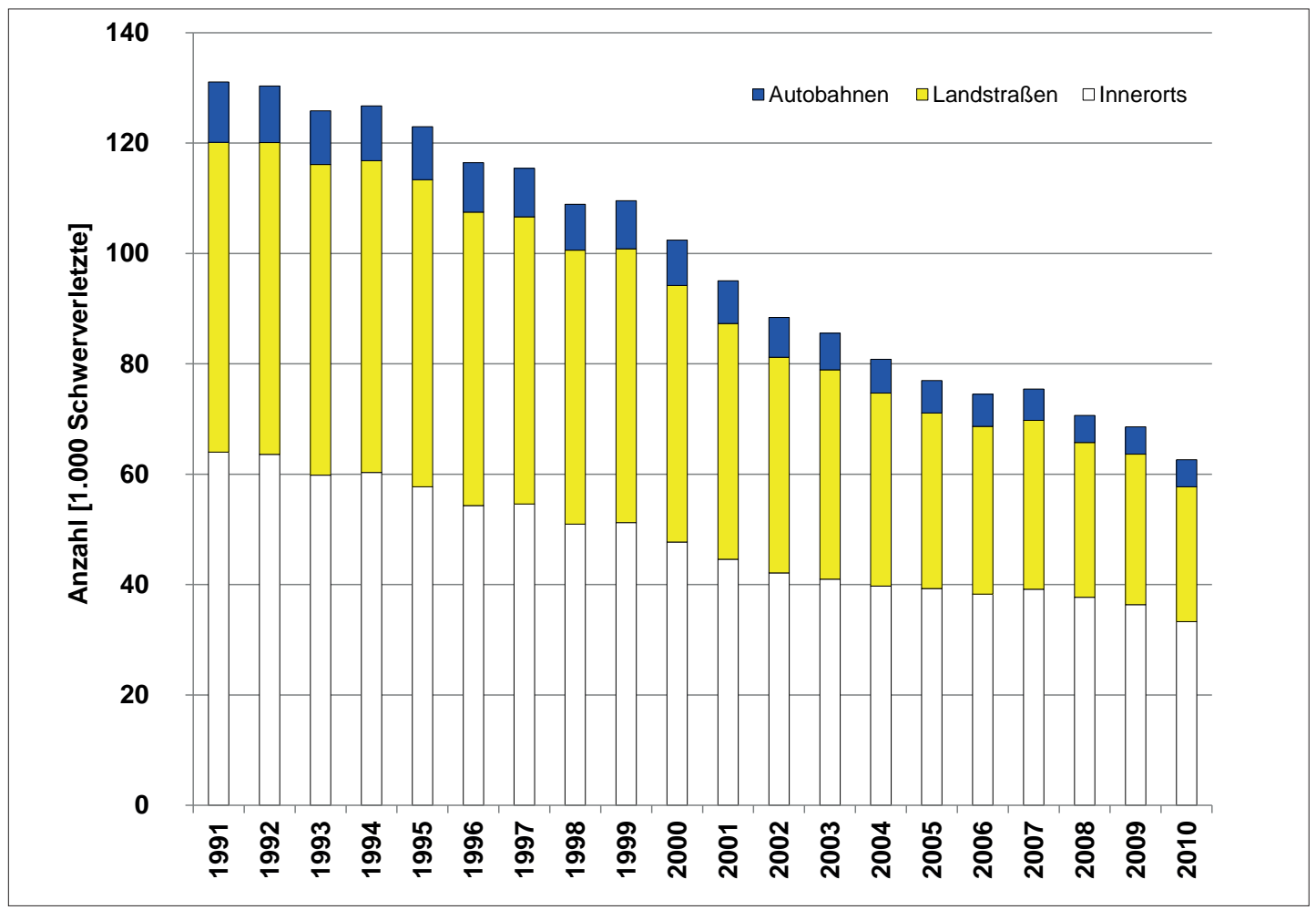

Abbildung $1 \mathrm{~b}$ :

Entwicklung von schweren Personenschäden (Schwerverletzte) nach Ortslage seit 1991

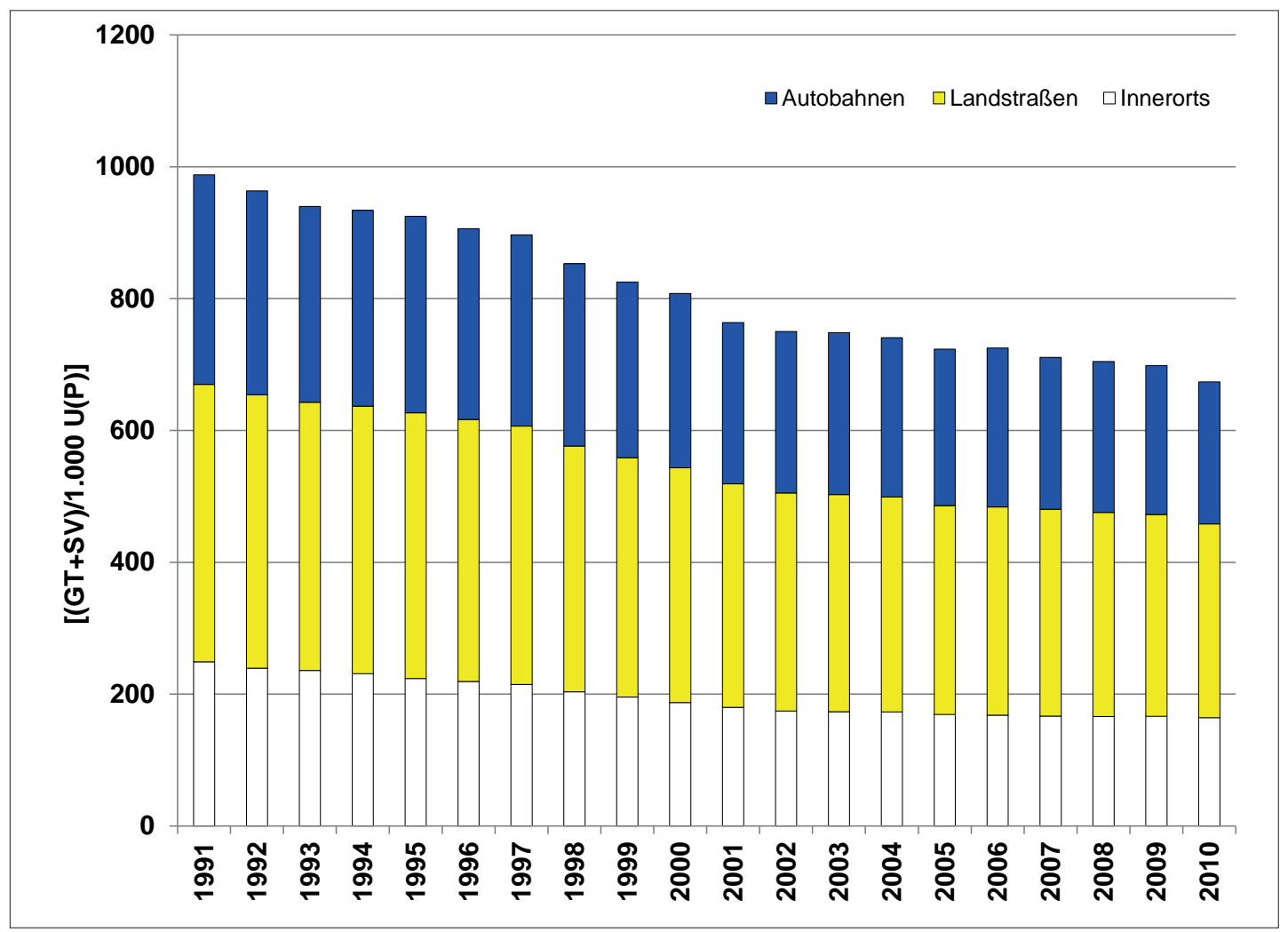

Abbildung $1 \mathrm{c}$ :

Entwicklung von schweren Personenschäden (Getötete und Schwerverletzte) nach Ortslage seit 1991 


\section{Vorgehensweise und Methodik}

Die Vorgehensweise bei der Untersuchung ist in Abbildung 2 dargestellt. Nachfolgend werden die einzelnen Arbeitsschritte kurz beschrieben.

\section{Literaturrecherche}

In einer umfangreichen Literaturrecherche wurden die auf dem Markt vorhandenen und die sich in der Entwicklung befindenden Fahrerassistenzsysteme analysiert, untereinander verglichen und in Bezug auf möglichen Nutzen für die Verkehrssicherheit überprüft. Dabei wurde zwischen vier verschiedenen Arten von Fahrerassistenzsystemen unterschieden:

- Infrastrukturautarke Systeme (z. B. Wechselverkehrszeichen),

- Fahrzeugautarke Systeme (z. B. Fahrdynamikregelungen),
- V2V-basierte Systeme (Fahrzeug zu Fahrzeug),

- V2I-basierte Systeme (Fahrzeug zu Infrastruktur und umgekehrt).

Ein kooperatives V2I-basiertes Fahrerassistenzsystem wird durch bidirektionale Kommunikation zwischen Infrastruktur und Fahrzeugen gekennzeichnet. Die berührungslose Erfassung von Verkehrsteilnehmern und Gefahren erfolgt mittels Sensoren, die sowohl in Fahrzeugen als auch seitens der Infrastruktur angebracht sein können.

Bei der Bearbeitung wurde insbesondere Wert auf die volkswirtschaftlich sinnvolle Umsetzbarkeit von V2I-basierten Fahrerassistenzsystemen gelegt. Als maßgebendes Kriterium wurde dabei die alternative Umsetzbarkeit durch fahrzeugautarke und V2V-basierten Fahrerassistenzsystemen verwendet.

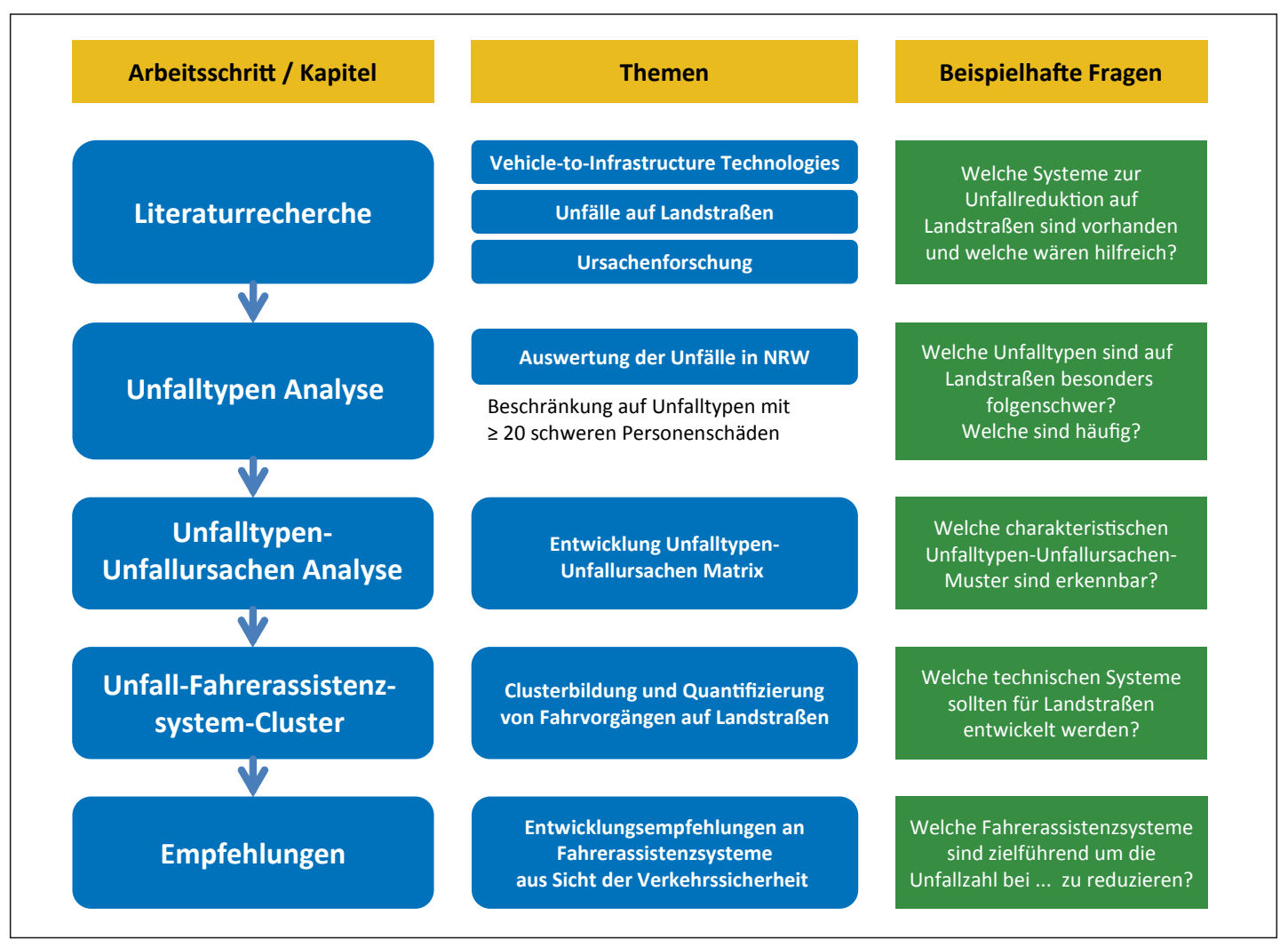

Abbildung 2:

Vorgehensweise und Arbeitsschwerpunkte des Projektes 


\section{Analyse der Unfalltypen}

Als Grundlage für die Ableitung von Anforderungen an technische Systeme aus Sicht der Verkehrssicherheit ist eine detaillierte Unfallstatistik erforderlich. Das Statistische Bundesamt veröffentlicht aggregierte Unfalldaten, welche anhand von sieben Unfalltypen (UT) aufgeschlüsselt sind. Allerdings sind die dabei verwendeten einstelligen Unfalltypen nicht detailliert genug, um umfassende Rückschlüsse auf den Unfallhergang zu ermöglichen. Beispielsweise sind Unfälle mit lichtsignalisierten Linksabbiegern anders zu betrachten als Unfälle mit Rechtsabbiegern, jedoch sind beide in den Unfalldaten vom Statistischen Bundesamt als Unfalltyp 2 „Abbiegeunfall“ enthalten.

Die Polizei in Nordrhein-Westfalen führt eine Unfalldatenbank, welche die sieben Unfalltypen in insgesamt 295 verschiedene, dreistellige Unfalltypen aufgliedert. Aufgrund dieser Detaillierung wurden für die Untersuchung anonymisierte Unfalldaten aus dieser Datenbank verwendet. Als Untersuchungszeitraum dienten die Jahre 2004 bis 2008. Innerhalb dieser Zeit ereigneten sich auf Landstraßen in Nordrhein-Westfalen 89.391 polizeilich registrierte Unfälle.

\section{Unfalltyp-Unfallursache-Analyse}

Um die Anzahl der zu berücksichtigenden Unfalltypen zu reduzieren, wurden seltene und/ oder weniger schwerwiegende Unfalltypen mit weniger als 20 schweren Personenschäden (SP) im fünfjährigen Untersuchungszeitraum ausgeschlossen. Der Schwellenwert wurde anhand einer Betrachtung der Summenkurve der Anzahl an schweren Personenschäden aller (absteigend sortierten) Unfalltypen festgelegt. Dadurch konnte die Anzahl der Unfalltypen von 295 auf 78 (um $74 \%$ ) gesenkt werden, während sich die Anzahl an Unfällen nur von 89.391 auf 84.405 (um 6 \%) verringerte. Dieser großen Anzahl an Unfällen wurden die zugehörigen Un-

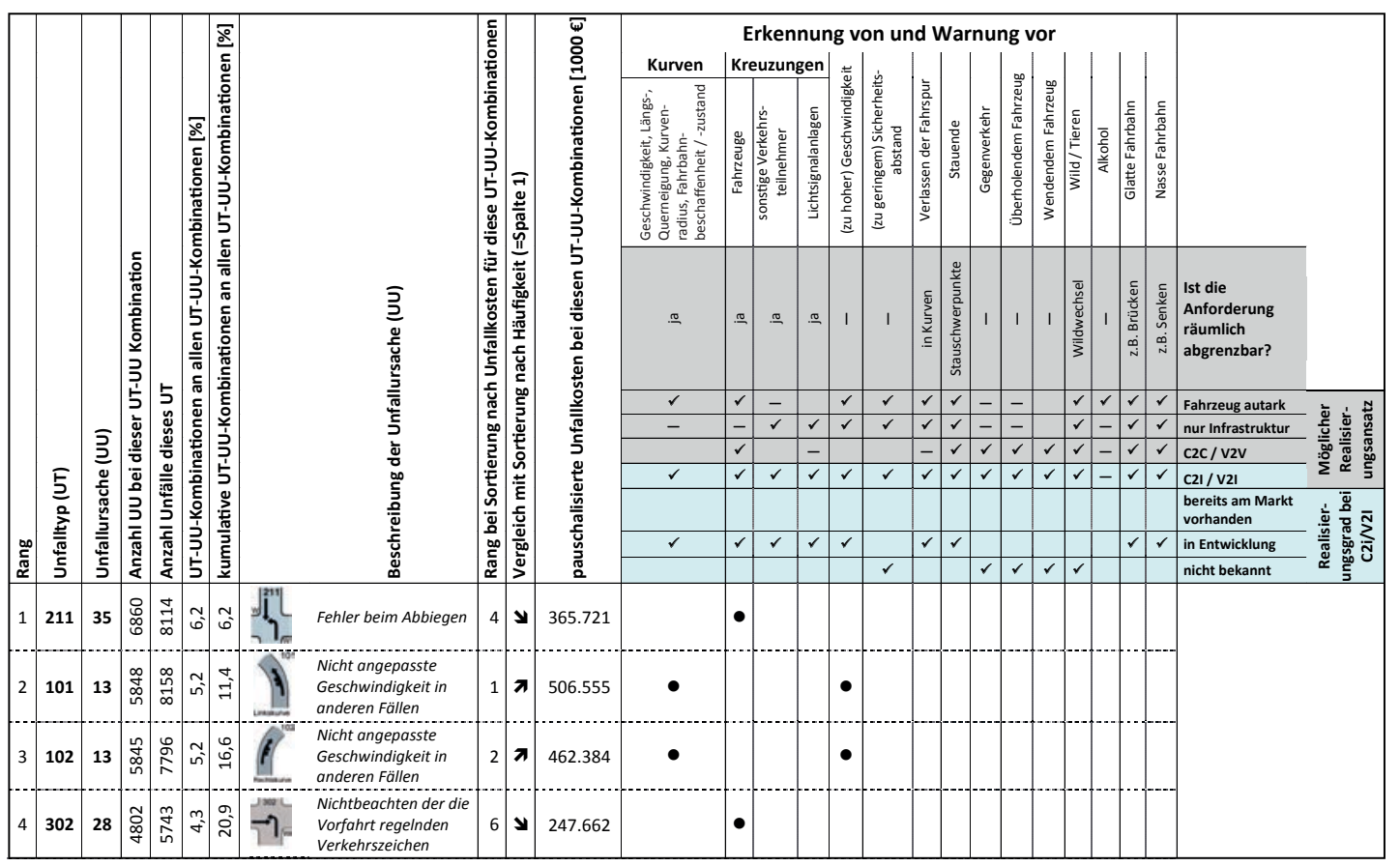

Abbildung 3:

Häufigste Unfalltyp-Unfallursachen-Kombinationen 
fallursachen (UU) gegenübergestellt. Hierfür wurden die 78 verbleibenden Unfalltypen mit den berichteten Unfallursachen kombiniert, die Unfallkenngrößen über alle zugehörigen Unfälle aggregiert und die 5.266 möglichen UT-UU-Kombinationen absteigend sortiert. Die vier häufigsten UT-UU-Kombinationen sind als Beispiele in Abbildung 3 dargestellt.

Diesen Kombinationen wurden Anforderungen an eine technische Realisierung gegenübergestellt (vgl. Abbildung 3). Bei Abbiegefehlern mit Kollision (Rang 1) muss z. B. ein geeignetes Fahrerassistenzsystem andere Fahrzeuge erkennen können. Bei Fahrunfällen in Kurven (Rang 2) müssen u.a. der Kurvenradius, die Fahrbahnbeschaffenheit und die Geschwindigkeit berücksichtigt werden.

\section{Unfall-Fahrerassistenzsystem-Cluster}

Basierend auf der Unfalltyp-Unfallursachen Analyse wurden Unfall-FahrerassistenzsystemCluster (UFASC) entwickelt. Diese bündeln fahrdynamisch ähnliche UT-UU-Kombinationen. Als Beispiel kann hier ein Fehler beim Abbiegen (UU 35) eines Linksabbiegers (UT 211) und ein Nichtbeachten der Vorfahrt regelnden Verkehrszeichen (UU 28) eines Linkseinbiegers (UT 302) (Abbildung 4) angeführt werden. In beiden Fällen ist der Fahrvorgang vergleichbar, da ein anderes Fahrzeug bei einem Fahrvorgang nach links übersehen oder falsch eingeschätzt wurde. Die Anforderungen an eine technische Realisierung sind somit gleich.

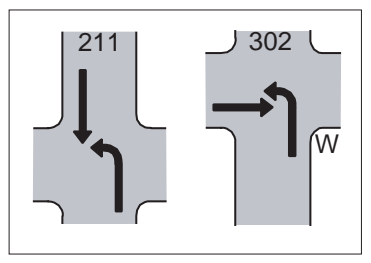

Abbildung 4: Unfalltypen 211 und 302

\section{Ergebnisse der Unfall-Fahrer- assistenzsystem-Cluster}

\subsection{Cluster - Übersicht}

Insgesamt wurden 20 verschiedene Fallgruppen detailliert untersucht. Bei der Entwicklung der Cluster wurden fahrdynamisch und fahraufgabenbezogen ähnliche Unfalltypen zusammengefasst. In der Folge ergeben sich räumliche und Unfallursachen-bezogene Ausprägungen bei den identifizierten Clustern. Diese sind als Übersicht in Tabelle 1 dargestellt.

Räumlich wurde dabei zwischen Unfällen auf freier Strecke (Gerade oder Kurve) und Unfällen an Knotenpunkten unterschieden. Des Weiteren wurden diverse Umfeldbedingungen näher untersucht. Aus Gründen der Vollständigkeit wurden alkoholisierte Fahrzeuglenker (UU 1) und ",andere Fehler beim Fahrer" (UU 49) ebenfalls behandelt.

Es wird darauf hingewiesen, dass ein einzelner Unfall in verschiedenen Clustern berücksichtigt sein kann; z. B. ist ein Unfall, der sich in einem Stau bei Nässe ereignet hat, unter „Unfällen bei Stau“ und „Unfällen bei Nässe“ enthalten.

Im Folgenden werden hinsichtlich der Vorfallrelevanz besonders auffällige Cluster näher beschrieben.

\subsection{Ausgewählte Cluster}

\subsubsection{Unfälle an Knotenpunkten}

\section{Beschreibung des Unfall-Fahrerassistenz- system-Clusters}

Dieses Cluster kombiniert Konfliktsituationen am Knotenpunkt mit und ohne Lichtsignalanlage (LSA) mit Fahrzeugen von links (UT 301, 
Tabelle 1:

Übersicht der untersuchten Unfall-Fahrerassistenzsystem-Cluster

\begin{tabular}{|c|c|c|c|c|c|c|c|}
\hline \multirow{2}{*}{\multicolumn{2}{|c|}{ Kurzform }} & \multirow[b]{2}{*}{ Beschreibung } & \multicolumn{5}{|c|}{ Rangfolge nach } \\
\hline & & & $\mathrm{U}$ & GT & $\mathrm{SP}$ & $\begin{array}{c}\text { SP/ } \\
1000 \mathrm{U}\end{array}$ & UK \\
\hline \multirow{3}{*}{ 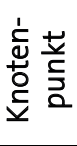 } & KP1 & Unfälle infolge von Vorfahrtsfehlern & 1 & 3 & 2 & 17 & 2 \\
\hline & KP2 & Unfälle bei eingeschalteter LSA & 4 & 13 & 9 & 19 & 9 \\
\hline & KP3 & Unfälle mit Fußgängern und Radfahrern & 8 & 5 & 7 & 5 & 7 \\
\hline \multirow{9}{*}{ 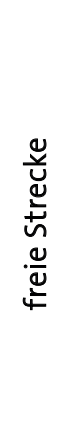 } & FS1 & Fahrunfälle in Kurven & 3 & 2 & 3 & 8 & 3 \\
\hline & FS2 & Fahrunfälle auf Geraden & 11 & 10 & 10 & 6 & 10 \\
\hline & FS3 & Unfälle bei Stau & 15 & 20 & 18 & 20 & 18 \\
\hline & FS4 & Unfälle bei überholenden Fahrzeugen & 17 & 14 & 15 & 15 & 15 \\
\hline & FS5 & Unfälle durch begegnende Fahrzeuge & 7 & 4 & 4 & 2 & 6 \\
\hline & FS6 & Unfälle bei wendenden Fahrzeugen & 14 & 16 & 16 & 18 & 16 \\
\hline & FS7 & Unfälle mit Fußgängern und Radfahrern & 13 & 9 & 13 & 1 & 13 \\
\hline & FS8 & Unfälle mit Tieren & 16 & 17 & 14 & 12 & 14 \\
\hline & FS9 & Unfälle mit temporären Hindernissen & 10 & 6 & 8 & 3 & 8 \\
\hline \multirow{6}{*}{ 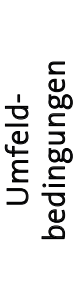 } & UB1 & Unfälle bei Nässe & 12 & 12 & 12 & 16 & 12 \\
\hline & UB2 & Unfälle bei Eis und Schnee & 9 & 11 & 11 & 13 & 11 \\
\hline & UB3 & Unfälle bei reduzierter Fahrbahngriffigkeit & 5 & 8 & 6 & 14 & 4 \\
\hline & UB4 & Unfälle bei Nebel & 19 & 18 & 19 & 11 & 19 \\
\hline & UB5 & Unfälle bei Seitenwind & 20 & 19 & 20 & 4 & 20 \\
\hline & UB6 & Unfälle bei blendender Sonne & 18 & 15 & 17 & 10 & 17 \\
\hline \multirow{2}{*}{$\frac{1}{2} \stackrel{0}{\frac{1}{4}}$} & UU1 & Unfälle mit alkoholisierten Beteiligten & 6 & 7 & 5 & 9 & 5 \\
\hline & UU49 & Unfälle mit „andere Fehler beim Fahrer“ & 2 & 1 & 1 & 7 & 1 \\
\hline
\end{tabular}

302, 303), von rechts (UT 321, 322) sowie mit Gegenverkehr bei Linksabbiegern (UT 211). Die gewählten Konfliktströme ließen sich prinzipiell mit einem eigensignalisierten Abbiegestrom sichern. Bei den Unfallursachen werden Missachtung der Verkehrsregelung (auch mit Lichtzeichen) (UU 27, 28 und 31), das Nichtbeachten entgegenkommender Fahrzeuge (UU 32) sowie Fehler beim Abbiegen (UU 35) betrachtet.

Die statistischen Angaben in Tabelle 2 beinhalten alle Ausprägungen des betrachteten Clusters. Bei einer zusätzlichen Einschränkung auf Unfälle an Lichtsignalanlagen konnte festgestellt werden, dass die Unfallschwere stark abnimmt.
Die relativen Angaben ermöglichen einen Vergleich der Folgenschwere zwischen verschiedenen Clustern. Im konkreten Beispiel der Unfälle an Knotenpunkten bedeutet dies, dass 29,2 \% aller Unfälle „nur“ 19,6 \% aller Unfälle mit Getöteten (Unfallkategorie 1) beinhalten. Somit ist dieses Cluster vergleichsweise weniger folgenschwer als der Durchschnitt. Bezogen auf die Beteiligten ist die Differenz zwischen Anteil Beteiligte und Anteil Getötete noch ausgeprägter. Die um etwa Faktor zwei erhöhte Anzahl an Beteiligten kann durch den definitionsbedingten Ausschluss an Alleinunfällen begründet werden (ein Alleinunfall am Knotenpunkt wäre als UT 121, 122, 123 klassifiziert). 


\section{Anforderungen an eine technische Umsetzung}

Ein Knotenpunktassistent benötigt möglichst genaue Positions- (z.B. Fahrspur), Bewegungs(Fahrtrichtung, Geschwindigkeit, Beschleunigung/Verzögerung) und weitere Zustandsdaten (z.B. Fahrtrichtungsanzeiger) möglichst aller Verkehrsteilnehmer im Vorfeld und unmittelbaren Bereich des Kontenpunktes sowie die Zustandsdaten vorhandener Lichtsignalanlagen, um eine wahrscheinlichkeitsbasierte Prognose der zukünftigen Bewegungen in einer dynamischen Karte zu berechnen. Übersteigt die errechnete Wahrscheinlichkeit für eine gefährliche Konfliktsituationen einen definierten Schwellenwert, werden die betroffenen Fahrzeuge gewarnt. Technisch denkbar sind auch automatisierte Eingriffe.

\section{Empfehlung}

V2I-basierte Fahrerassistenzsysteme sind an unfallträchtigen, lichtsignalisierten Knotenpunkten zu empfehlen, da sie hier aufgrund der möglichen Anpassungen an die örtlichen Gegebenheiten (z. B. bei Sichtbehinderungen durch Bebauungen) und der hierarchischen Steuerung komplexer Verkehrssituationen fahrzeugautarken und V2V-basierten Systemen überlegen sind.

\subsubsection{Fahrunfälle in Kurven und auf Geraden mit unangepasster Geschwindigkeit}

\section{Beschreibung des Unfall-Fahrerassistenz- system-Clusters}

Fahrunfälle in Kurven sind in der Regel besonders folgenschwer. Wie aus Tabelle 3 ersichtlich

Tabelle 2:

Statistischer Steckbrief für Unfälle infolge von Vorfahrtsfehlern mit und ohne Lichtsignalanlagen

\begin{tabular}{|c|c|c|c|c|c|c|}
\hline & \multicolumn{2}{|c|}{ Bedingungen } & & & & \\
\hline UT & \multicolumn{2}{|c|}{$\begin{array}{c}211,281,301,302 \\
303,321,322\end{array}$} & $\begin{array}{c}7 \text { UT } \\
\text { von } 77 \text { UT }\end{array}$ & & & \\
\hline UU & \multicolumn{2}{|c|}{$27,28,31,32,35$} & $\begin{array}{c}5 \text { UU } \\
\text { von } 69 \text { UU }\end{array}$ & & & \\
\hline $\begin{array}{c}\text { Weitere } \\
\text { Bedingungen }\end{array}$ & \multicolumn{3}{|c|}{ keine } & & & \\
\hline \multicolumn{4}{|l|}{ Cluster Statistik } & & & \\
\hline \multicolumn{3}{|c|}{$\begin{array}{c}\text { Beteiligtenbezogene } \\
\text { Betrachtungsweise }\end{array}$} & \multicolumn{4}{|c|}{ Unfallbezogene Betrachtungsweise } \\
\hline & Anzahl & Anteil & & Anzahl & Anteil & Unfallkosten \\
\hline Beteiligte & 50788 & $33,9 \%$ & Unfälle & 24613 & $29,2 \%$ & $1.539 .561 .000 €$ \\
\hline Getötete & 360 & $18,8 \%$ & Unfallkategorie 1 & 344 & $19,6 \%$ & $92.880 .000 €$ \\
\hline Schwerverletzte & 5416 & $24,5 \%$ & Unfallkategorie 2 & 4202 & $23,4 \%$ & $1.134 .540 .000 €$ \\
\hline \multirow[t]{2}{*}{ Leichtverletzte } & \multirow{2}{*}{\multicolumn{2}{|c|}{$28,7 \%$}} & Unfallkategorie 3 & 10254 & $24,4 \%$ & $184.572 .000 €$ \\
\hline & & & Unfallkategorie 4 & 9813 & $43,1 \%$ & $127.569 .000 €$ \\
\hline Verkehrsmodi & Anzahl & Anteil & Unfälle auf: & Anzahl & Anteil & Unfallkosten \\
\hline Pkw & 42141 & $37,1 \%$ & Bundesstraßen & 6987 & $30,0 \%$ & $437.284 .000 €$ \\
\hline Bus & 191 & $27,1 \%$ & Landesstraßen & 11946 & $31,3 \%$ & $745.821 .000 €$ \\
\hline Lkw & 3189 & $32,5 \%$ & Kreisstraßen & 3478 & $25,5 \%$ & $231.783 .000 €$ \\
\hline Motorrad & 3518 & $25,9 \%$ & sonstigen Straßen & 2202 & $23,6 \%$ & $124.673 .000 €$ \\
\hline Fußgänger & 18 & $0,9 \%$ & & & & \\
\hline Fahrrad & 1222 & $16,6 \%$ & & & & \\
\hline Sonstige & 509 & $21,0 \%$ & & & & \\
\hline
\end{tabular}


ist, führten Fahrunfälle in Kurven mit nicht angepasster oder überhöhter Geschwindigkeit zu rund einem Viertel aller Getöteten und mehr als einem Fünftel aller Schwerverletzten auf Landstraße.

Fahrunfälle auf Geraden ereigneten sich seltener, sind jedoch ähnlich folgenschwer (Tabelle 4). Sehr viele Unfälle auf Geraden werden mit der Unfallursache „andere Fehler beim Fahrer" versehen, welche in dem in Tabelle 4 aufgeführten Cluster nicht berücksichtigt wird.

\section{Anforderungen an eine technische Umsetzung}

Ein Fahrerassistenzsystem muss Fahrzeuge mit nicht angepasster Geschwindigkeit rechtzeitig erkennen (z. B. vereinfacht durch die Überschreitung der zulässigen Höchstge- schwindigkeit oder komplexer unter Berücksichtigung weiterer Rahmenbedingungen wie Witterung, Reifenhaftung, Fahrbahngriffigkeit etc.) und warnen, um entsprechende Unfälle zu vermeiden.

\section{Empfehlung}

Hier sind fahrzeugautarke Fahrerassistenzsysteme zu bevorzugen, da sie bei ausgestatteten Fahrzeugen potentiell in allen Kurven und auf allen Geraden wirken könnten. Da die wichtigsten Eingangsgrößen statisch (z. B. Straßengeometrie) oder relativ leicht zu erfassen sind (z. B. Geschwindigkeit), könnte die Warnung zur schnelleren Marktdurchdringung in mobile Anwendungen wie z. B. Navigationssysteme oder Smartphones integriert werden.

Tabelle 3:

Statistischer Steckbrief für Fahrunfälle in Kurven

\begin{tabular}{|c|c|c|c|c|c|c|c|}
\hline & \multicolumn{3}{|c|}{ Bedingungen } & & & & \\
\hline UT & \multicolumn{3}{|c|}{$\begin{array}{c}101,102,121,122,123 \\
131,132,151,152 \\
\end{array}$} & $\begin{array}{c}9 \text { UT } \\
\text { von } 77 \text { UT }\end{array}$ & & & \\
\hline UU & \multicolumn{3}{|c|}{12,13} & $\begin{array}{c}2 \mathrm{UU} \\
\text { von } 69 \mathrm{UU}\end{array}$ & & & \\
\hline $\begin{array}{c}\text { Weitere } \\
\text { Bedingungen }\end{array}$ & \multirow{2}{*}{\multicolumn{4}{|c|}{ Keine }} & \multirow{2}{*}{\multicolumn{3}{|c|}{$\begin{array}{l}\text { Fahrunfälle in Kurve (inkl. Steigungen usw. ) } \\
\text { mit nicht angepasster Geschwindigkeit }\end{array}$}} \\
\hline Cluster Statistik & & & & & & & \\
\hline \multicolumn{3}{|c|}{$\begin{array}{c}\text { Beteiligtenbezogene } \\
\text { Betrachtungsweise }\end{array}$} & \multicolumn{5}{|c|}{ Unfallbezogene Betrachtungsweise } \\
\hline & Anzahl & Anteil & & & Anzahl & Anteil & Unfallkosten \\
\hline Beteiligte & 18910 & $12,6 \%$ & & Unfälle & 15907 & $18,8 \%$ & $1.357 .966 .000 €$ \\
\hline Getötete & 482 & $25,2 \%$ & \multicolumn{2}{|c|}{ Unfallkategorie 1} & 435 & $24,7 \%$ & $117.450 .000 €$ \\
\hline Schwerverletzte & 4776 & $21,6 \%$ & \multicolumn{2}{|c|}{ Unfallkategorie 2} & 3915 & $21,8 \%$ & $1.057 .050 .000 €$ \\
\hline Leichtverletzte & 9466 & $14,7 \%$ & \multicolumn{2}{|c|}{ Unfallkategorie 3} & 6645 & $15,8 \%$ & $119.610 .000 €$ \\
\hline & & & \multicolumn{2}{|c|}{ Unfallkategorie 4} & 4912 & $21,6 \%$ & $63.856 .000 €$ \\
\hline Verkehrsmodi & Anzahl & Anteil & \multicolumn{2}{|c|}{ Unfälle auf: } & Anzahl & Anteil & Unfallkosten \\
\hline Pkw & 15203 & $13,4 \%$ & \multicolumn{2}{|c|}{ Bundesstraßen } & 3221 & $13,8 \%$ & $249.601 .000 €$ \\
\hline Bus & 53 & $7,5 \%$ & \multicolumn{2}{|c|}{ Landesstraßen } & 7584 & $19,9 \%$ & $653.309 .000 €$ \\
\hline Lkw & 849 & $8,6 \%$ & \multicolumn{2}{|c|}{ Kreisstraßen } & 3299 & $24,2 \%$ & $312.454 .000 €$ \\
\hline Motorrad & 2470 & $18,2 \%$ & \multicolumn{2}{|c|}{ sonstigen Straßen } & 1803 & $19,4 \%$ & $142.602 .000 €$ \\
\hline Fußgänger & 61 & $2,9 \%$ & & & & & \\
\hline Fahrrad & 127 & $1,7 \%$ & & & & & \\
\hline Sonstige & 147 & $6,1 \%$ & & & & & \\
\hline
\end{tabular}


Tabelle 4:

Statistischer Steckbrief für Fahrunfälle auf Geraden

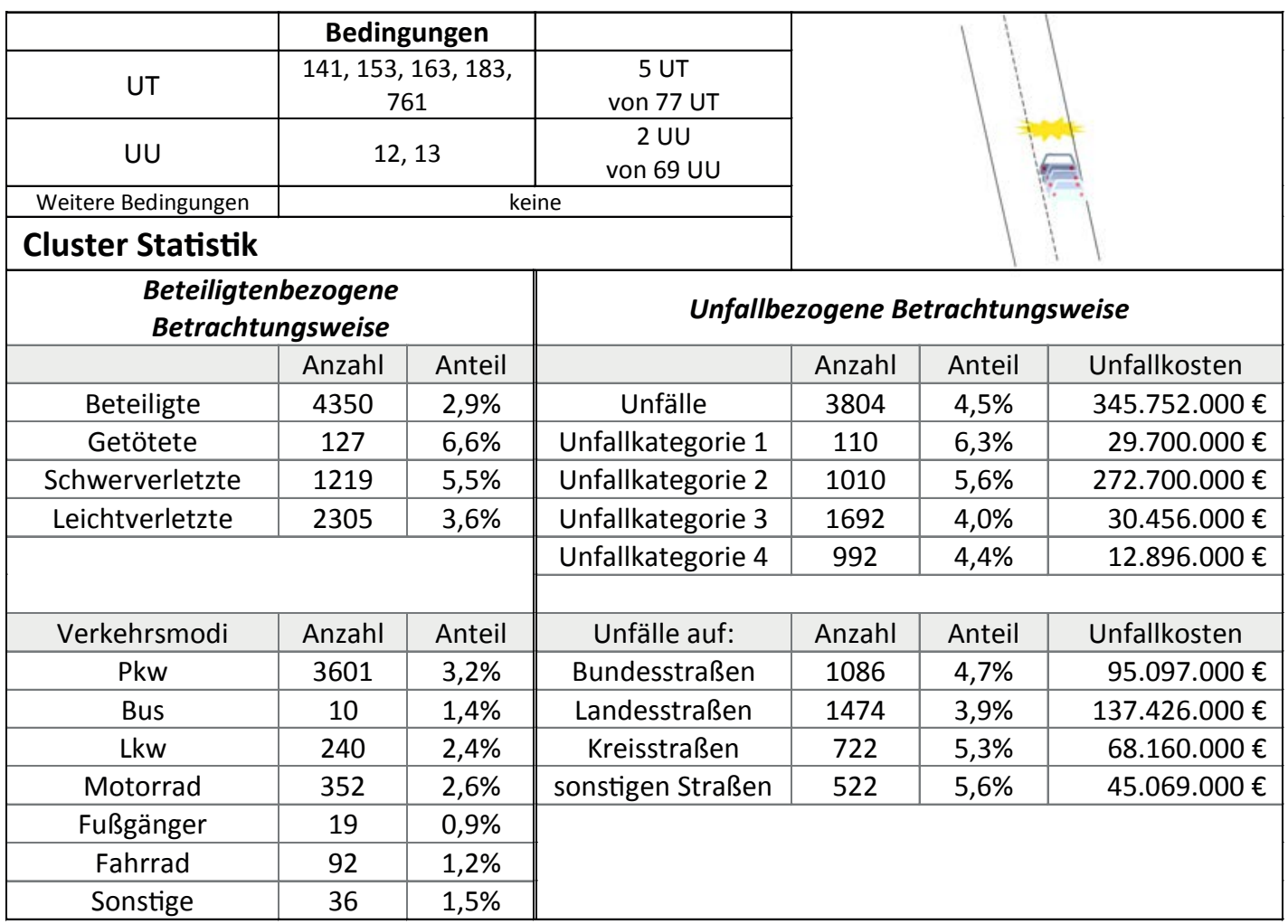

Infrastrukturautarke Systeme mit Geschwindigkeitsmessung und Wechselverkehrszeichen vor unfallträchtigen Kurven, könnten Fahrer von nicht ausgestatteten Fahrzeugen auf nicht angepasste Geschwindigkeiten hinweisen.

V2I-basierte Systeme sind in diesem Cluster wenig zielführend, da sie nur punktuell und für Fahrer mit ausgestatteten Fahrzeugen wirken.

\subsubsection{Unfälle mit Gegenverkehr}

\section{Beschreibung des Unfall-Fahrerassistenz- system-Clusters}

Das in Tabelle 5 skizzierte Cluster setzt sich aus Unfällen bei Gegenverkehr zusammen. Dies sind sowohl Unfälle beim Überholen (UT 661) als auch beim Begegnen auf Geraden (UT 681) und in Kurven (UT 682). Da die drei verwendeten Unfalltypen sehr präzise abgegrenzt sind, wurde auf eine besondere Selektion bestimmter Unfallursachen verzichtet.

\section{Anforderungen an eine technische Umsetzung}

Die Mindestanforderung an ein geeignetes Assistenzsystem sollte sein, den Fahrer bei unbeabsichtigtem Verlassen des Fahrstreifens zu warnen. Dies ist am einfachsten über eine Erkennung der Fahrbahnmarkierungen zu realisieren, wie es bereits heute mit fahrzeugautarken Spurassistenten geschieht. Diesem System sind jedoch Grenzen gesetzt, da abgefahrene, überwachsene oder verschmutzte Markierungen nicht oder nur unzureichend erkannt werden können.

Wesentlich komplexer wäre ein System, das u.a. die hochgenauen Positionen, Fahrtrichtungen und Geschwindigkeiten sämtlicher relevanter Fahrzeuge erfasst, um daraus deren 
Tabelle 5:

Statistischer Steckbrief für „Unfälle bei Gegenverkehr“

\begin{tabular}{|c|c|c|c|c|c|c|}
\hline & \multicolumn{3}{|c|}{ Bedingungen } & & \multirow{5}{*}{9} & \\
\hline UT & \multicolumn{2}{|c|}{$661,681,682$} & $\begin{array}{c}3 \text { UT } \\
\text { von } 77 \text { UT }\end{array}$ & & & \\
\hline UU & \multicolumn{2}{|r|}{ alle } & $\begin{array}{c}69 \text { UU } \\
\text { von } 69 \text { UU }\end{array}$ & & & \\
\hline $\begin{array}{c}\text { Weitere } \\
\text { Bedingungen }\end{array}$ & \multirow{2}{*}{\multicolumn{3}{|c|}{ keine }} & & & \\
\hline \multicolumn{3}{|l|}{ Cluster Statistik } & & & & \\
\hline \multicolumn{3}{|c|}{$\begin{array}{c}\text { Beteiligtenbezogene } \\
\text { Betrachtungsweise }\end{array}$} & \multicolumn{4}{|c|}{ Unfallbezogene Betrachtungsweise } \\
\hline & Anzahl & Anteil & & Anzahl & Anteil & Unfallkosten \\
\hline Beteiligte & 11572 & $7,7 \%$ & Unfälle & 5339 & $6,3 \%$ & $486.985 .000 €$ \\
\hline Getötete & 265 & $13,9 \%$ & Unfallkategorie 1 & 232 & $13,2 \%$ & $62.640 .000 €$ \\
\hline Schwerverletzte & 2121 & $9,6 \%$ & Unfallkategorie 2 & 1347 & $7,5 \%$ & $363.690 .000 €$ \\
\hline \multirow[t]{2}{*}{ Leichtverletzte } & 4512 & \multirow[t]{2}{*}{$7,0 \%$} & Unfallkategorie 3 & 2355 & $5,6 \%$ & $42.390 .000 €$ \\
\hline & \multirow{2}{*}{ Anzahl } & & Unfallkategorie 4 & 1405 & $6,2 \%$ & $18.265 .000 €$ \\
\hline Verkehrsmodi & & Anteil & Unfälle auf: & Anzahl & Anteil & Unfallkosten \\
\hline Pkw & 8371 & $7,4 \%$ & Bundesstraßen & 1258 & $5,4 \%$ & $143.122 .000 €$ \\
\hline Bus & 121 & $17,1 \%$ & Landesstraßen & 2285 & $6,0 \%$ & $207.519 .000 €$ \\
\hline Lkw & 1251 & $12,7 \%$ & Kreisstraßen & 923 & $6,8 \%$ & $83.142 .000 €$ \\
\hline Motorrad & 741 & $5,4 \%$ & & \begin{tabular}{l|l} 
sonstigen Straßen & 873 \\
\end{tabular} & $9,4 \%$ & $53.202 .000 €$ \\
\hline Fußgänger & 20 & $1,0 \%$ & & & & \\
\hline Fahrrad & 738 & $10,0 \%$ & & & & \\
\hline Sonstige & 330 & $13,6 \%$ & & & & \\
\hline
\end{tabular}

zukünftige Bewegungen (Trajektorien) zu prognostizieren und somit mögliche Kollisionen rechtzeitig zu erkennen.

\section{Empfehlungen}

Fahrzeugautarke Spurassistenten können bei entsprechenden Randbedingungen Unfälle durch sich begegnende Fahrzeuge reduzieren. Eine Weiterentwicklung mit V2V-Kommunikation könnte vor entgegenkommenden Fahrzeugen auch ohne Sichtkontakt warnen, sofern diese ebenfalls ausgestattet sind. V2I-basierte Fahrerassistenzsysteme wären sehr aufwendig zu realisieren und in ihrer Wirkung lokal begrenzt. Sie sind daher eher ungeeignet, um Unfälle mit begegnenden Fahrzeugen zu reduzieren.

\subsubsection{Unfälle mit Fußgängern und Radfah- rern im Längsverkehr}

\section{Beschreibung des Unfall-Fahrerassistenz- system-Clusters}

In diesem Cluster werden nicht motorisierte Verkehrsteilnehmer betrachtet, die auf Landstraßen im Längsverkehr verunglückt sind. Für Unfälle mit Fußgängern sind z. T. explizite Unfalltypen vorhanden. Da es für Unfälle mit Radfahrern keinen eigenen Unfalltyp gibt, wurde die Mehrzahl der Unfälle über die Verkehrsbeteiligung von Fußgängern oder Radfahrern eingeschlossen. Deshalb sind auch Unfalltypen wie Fahrunfälle auf Geraden (UT 141) enthalten. 
Das Cluster zeichnet sich durch eine hohe Folgenschwere der Unfälle aus (vgl. Tabelle 6).

Bei einer Unterscheidung nach Lichtverhältnissen konnte festgestellt werden, dass Unfälle sich häufig bei Tageslicht ereignen, jedoch der Anteil an Getöteten bei Dunkelheit besonders hoch ist.

\section{Anforderungen an eine technische Umsetzung}

Obwohl bereits fahrzeugautarke Systeme am Markt sind, welche nicht motorisierte Verkehrsteilnehmer bei Dunkelheit erkennen kön- nen, kann aus der Untersuchung abgeleitet werden, dass ein geeignetes Fahrerassistenzsystem auch bei Tageslicht funktionieren sollte. Es sollte die Positionen von Fußgängern und Radfahrern sowohl bei Tageslicht als auch bei Nacht erkennen können und diese entsprechend (visuell) kennzeichnen.

\section{Empfehlungen}

Infrastrukturautarke Systeme, welche Fußgänger und Radfahrer im Längsverkehr detektieren und Fahrer anhand von Wechselverkehrszeichen darauf aufmerksam machen, könnten

Tabelle 6:

Statistischer Steckbrief für Unfälle mit Fußgängern und Radfahrern im Längsverkehr

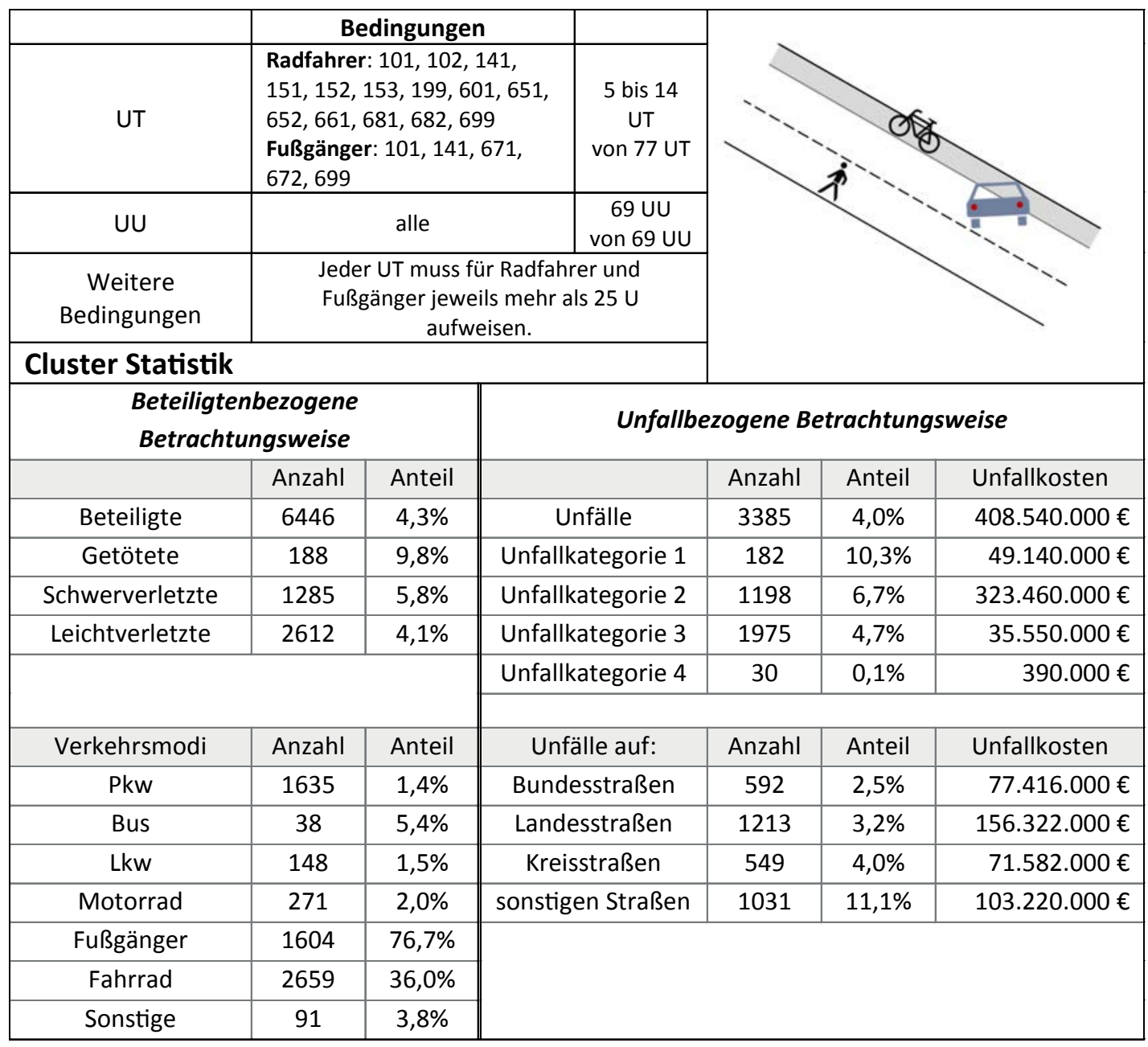


auf Landstraßenabschnitten mit einer entsprechenden Unfallhäufung installiert werden.

Die bestehenden, fahrzeugautarken Systeme sollten auch bei Tageslicht Fußgänger und Radfahrer erkennen können. Durch die V2VKommunikation sind nur geringe Vorteile zu erwarten. Auch V2I-basierte Fahrerassistenzsysteme sind wenig erfolgsversprechend, da sich die Unfälle räumlich dispers ereignen und daher mit hohen Installationskosten zu rechnen wäre.

Aufgrund der Unfallverteilung und der Unfallschwere ist die Weiterentwicklung und Verbreitung fahrzeugautarker Systeme zu forcieren, da sie flächenhaft wirken.

\subsection{Zielführende Lösungswege für Fahrerassistenzsysteme}

Tabelle 7 fasst die in der Untersuchung ermittelten zielführenden und weniger zielführenden Lösungswege für aus Sicht der Verkehrssicherheit relevante Fahrerassistenzsysteme zusammen. Die Untersuchung zeigt, dass die Vorteile von V2I-basierten Systemen insbesondere in komplexen, räumlich begrenzten (punktförmigen) Verkehrssituationen, wie z. B. an Knotenpunkten, zielführend eingesetzt werden können.

Plötzlich und räumlich dispers auftretende Ereignisse, wie z. B. verlorene Ladung oder wendende Fahrzeuge können am besten durch fahrzeugbasierte Systeme abgedeckt werden.

\section{Tabelle 7:}

Überblick an zielführenden Lösungswegen für Assistenzsysteme zur Erhöhung der Verkehrssicherheit

\begin{tabular}{|c|c|c|c|c|c|c|c|}
\hline $\begin{array}{l}\text { Cluster } \\
\text { Typ }\end{array}$ & \multicolumn{2}{|r|}{ Cluster } & $\begin{array}{l}2 \\
0 \\
8 \\
0 \\
\text { 김 }\end{array}$ & 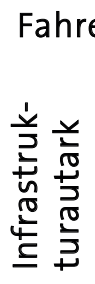 & 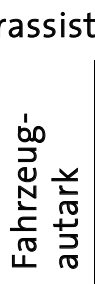 & ঐ & $\overline{\mathrm{N}}$ \\
\hline \multirow{3}{*}{$\begin{array}{l}\text { Knoten- } \\
\text { punkt }\end{array}$} & KP1 & Unfälle infolge von Vorfahrtsfehlern & 234,7 & $x$ & - & $\bullet$ & $\checkmark$ \\
\hline & KP2 & Unfälle bei eingeschalteter LSA & 161,9 & $x$ & $\bullet$ & $\bullet$ & $\checkmark$ \\
\hline & KP3 & $\begin{array}{l}\text { Unfälle mit Fußgängern und } \\
\text { Radfahrern }\end{array}$ & 369,6 & $\bullet$ & $\bullet$ & $\bullet$ & $\checkmark$ \\
\hline \multirow{9}{*}{$\begin{array}{l}\text { freie } \\
\text { Strecke }\end{array}$} & FS1 & Fahrunfälle in Kurven & 338,0 & $\checkmark$ & $\checkmark$ & $x$ & - \\
\hline & FS2 & Fahrunfälle auf Geraden & 352,3 & $x$ & $\checkmark$ & $x$ & $x$ \\
\hline & FS3 & Unfälle bei Stau & 112,2 & $x$ & $\bullet$ & $\checkmark$ & $x$ \\
\hline & FS4 & Unfälle bei überholenden Fahrzeugen & 250,0 & $x$ & $\bullet$ & $\checkmark$ & $x$ \\
\hline & FS5 & $\begin{array}{l}\text { Unfälle durch begegnende } \\
\text { Fahrzeuge }\end{array}$ & 446,9 & $x$ & $\bullet$ & $\checkmark$ & $x$ \\
\hline & FS6 & Unfälle bei wendenden Fahrzeugen & 213,4 & $x$ & - & $\sqrt{ }$ & $x$ \\
\hline & FS7 & $\begin{array}{l}\text { Unfälle mit Fußgängern und } \\
\text { Radfahrern }\end{array}$ & 523,8 & $x$ & $\checkmark$ & & $x$ \\
\hline & FS8 & Unfälle mit Tieren & 268,3 & $\checkmark$ & $\checkmark$ & & $\bullet$ \\
\hline & FS9 & Unfälle mit temporären Hindernissen & 435,2 & $x$ & $\checkmark$ & $x$ & $x$ \\
\hline $\begin{array}{l}\text { Umfeld- } \\
\text { bedin- } \\
\text { gungen }\end{array}$ & UB & $\begin{array}{l}\text { Unfälle aufgrund von Regen, Eis, } \\
\text { Nässe, usw. }\end{array}$ & 257,6 & $\bullet$ & $\checkmark$ & $\bullet$ & $x$ \\
\hline Sonstige & UU1 & $\begin{array}{l}\text { Unfälle mit alkoholisierten } \\
\text { Beteiligten }\end{array}$ & 328,5 & $x$ & $\checkmark$ & $x$ & $x$ \\
\hline
\end{tabular}




\section{Ergebnisse und Zusammen- fassung}

Tabelle 8 beschreibt die verschiedenen Abhängigkeiten und Ausprägungen von Fahrerassistenzsystemen. Heutige infrastrukturautarke Systeme (z. B. Lichtsignalanlagen, Streckenbeeinflussungsanlagen) werden von der öffentlichen Hand finanziert und sind für alle Fahrer nutzbar. Fahrzeugbasierte Fahrerassistenzsysteme (z. B. Spurassistent, Abstandstempomat) werden durch den Erwerb des Fahrzeugs finanziert und sind "nur" individuell nutzbar. Eine Sonderrolle ist V2I-basierten Fahrerassistenzsystemen beizumessen, da diese „nur“ individuell wirksam sind, jedoch nach heutigem Verständnis zu erheblichen Teilen durch die öffentliche Hand finanziert werden müssten.

Es sei an dieser Stelle darauf hingewiesen, dass die Finanzierung für die technische Funktionalität unwesentlich ist, die Kosten allerdings einen wesentlichen Einfluss auf die Verbreitung und somit Wirksamkeit derartiger Systeme haben. Vorstellbar wäre ebenfalls eine dauerhafte oder zeitlich befristete privatwirtschaftlich finanzierte, V2I-basierte Lösung, um entsprechend hohe Ausstattungsgrade und Wirkungen zu erzielen.

\section{Empfehlungen}

Die Untersuchung kommt zu dem Ergebnis, dass Fahrerassistenzsysteme (FAS) grundsätzlich einen großen Beitrag zur Erhöhung der Verkehrssicherheit leisten können. Die V2Ibasierte Variante ist jedoch wegen der zu installierenden, aufwendigen Technik räumlich beschränkt und wird daher nur punktuell einen relevanten Mehrwert zur Verkehrssicherheit leisten können.

Insgesamt wird aus Sicht der Verkehrssicherheit empfohlen, jene Systeme zu forcieren bzw. weiter zu entwickeln, die gegen nachfolgende häufige und folgenschwere Unfälle wirken:

- Unfälle auf Geraden infolge nicht angepasster Geschwindigkeit,

- Unfälle in Kurven infolge nicht angepasster Geschwindigkeit,

- Unfälle durch Ablenkung des Fahrers,

- Unfälle durch alkoholisierte Fahrzeuglenker.

Als „selten wirksam“ wurden diejenigen Systeme herausgearbeitet, die nur gegen selten auftretende oder wenig folgenschwere Unfälle wirken:

- Unfälle bei Nebel,

- Unfälle durch verlorene Ladung,

- Unfälle durch stehendes Fahrzeug.

Tabelle 8:

Vergleich der verschiedenen Arten von Assistenzsystemen

\begin{tabular}{|c|c|c|c|c|}
\hline & $\begin{array}{c}\text { Träger / } \\
\text { Verantwortlicher }\end{array}$ & $\begin{array}{c}\text { Kommunikation } \\
\text { notwendig }\end{array}$ & \multicolumn{2}{|c|}{ Wirksamkeit / Sichtbarkeit } \\
\cline { 4 - 5 } $\begin{array}{c}\text { Infrastruktur- } \\
\text { autarke Systeme }\end{array}$ & $\begin{array}{c}\text { öffentlicher } \\
\text { Baulastträger }\end{array}$ & & $\checkmark$ & \\
\hline $\begin{array}{c}\text { Fahrzeugautarke } \\
\text { Systeme }\end{array}$ & Fahrzeugindustrie & & & $\checkmark$ \\
\hline $\begin{array}{c}\text { V2l-basierende } \\
\text { Systeme }\end{array}$ & $\begin{array}{c}\text { vorrangig } \\
\text { Öffentlicher } \\
\text { Baulastträger }\end{array}$ & $\checkmark$ & & $\checkmark$ \\
\hline $\begin{array}{c}\text { V2V-basierende } \\
\text { Systeme }\end{array}$ & Fahrzeugindustrie & $\checkmark$ & & $\checkmark$ \\
\hline
\end{tabular}


V2I-basierte Fahrerassistenzsysteme könnten insbesondere an Knotenpunkten zum Einsatz kommen. In kürzlich abgeschlossenen Forschungsprojekten wie SAFESPOT und InterSafe2 konnte nachgewiesen werden, dass eine technische Umsetzung bereits heute möglich ist. Ungeklärt sind bislang jedoch die Wirksamkeit, Standardisierung, Betrieb, Haftung und insbesondere die Finanzierung der auf Seiten der Infrastruktur erforderlichen Technik. 


\section{GDV}

DIE DEUTSCHEN VERSICHERER

Gesamtverband der Deutschen Versicherungswirtschaft e.V.

Wilhelmstraße 43/43G, 10117 Berlin

Postfach 0802 64, 10002 Berlin

Tel.: 030/20 20 - 50 00, Fax: 030/2020-6000

www.gdv.de, www.udv.de 